\title{
The Vertical and Horizontal Spatial-Temporal Conceptual Metaphor Representation of Chinese Temporal Words
}

\author{
Tian Hong1, Xianyou He ${ }^{1 *}$, Richard Tillman'2, Xueru Zhao1, Yumei Deng³ \\ ${ }^{1}$ Guangdong Key Laboratory of Mental Health and Cognitive Science, Center for Studies of Psychological Application, School of \\ Psychology, South China Normal University, Guangzhou, China \\ ${ }^{2}$ Tilburg Center for Cognition and Communication, Tilburg University, Tilburg, Netherlands \\ ${ }^{3}$ Guangdong Institute of Public Administration, Guangzhou, China \\ Email: *xianyouhe@163.com
}

How to cite this paper: Hong, T., He, $\mathrm{X}$. Y., Tillman, R., Zhao, X. R., \& Deng, Y. M. (2017). The Vertical and Horizontal Spatial-Temporal Conceptual Metaphor Representation of Chinese Temporal Words. Psychology, 8, 1679-1692.

https://doi.org/10.4236/psych.2017.811111

Received: July 27, 2017

Accepted: September 1, 2017

Published: September 4, 2017

Copyright $\odot 2017$ by authors and Scientific Research Publishing Inc. This work is licensed under the Creative Commons Attribution International License (CC BY 4.0).

http://creativecommons.org/licenses/by/4.0/

\begin{abstract}
People rely on spatial-temporal metaphor when they talk and think about abstract temporal concept. The purpose of this study is to further investigate the mechanism of the multi-dimensional spatial-temporal conceptual metaphor in Chinese. Using the spatial cuing paradigm, we examined the cognitive impact of the Chinese vertical and horizontal spatial-temporal metaphor, and explored the dominant dimension between the two metaphors in context. The results showed that the processing of temporal concepts for Chinese speakers involved not only the horizontal spatial-temporal metaphor, but also the vertical spatial-temporal metaphor. The horizontal dimension was the dominant dimension of spatial-temporal metaphor in the processing of temporal concepts in Chinese. The findings demonstrated that representation of time depends on representation of space, supporting the Metaphorical Structuring View.
\end{abstract}

\section{Keywords}

Spatial-Temporal Conceptual Metaphor, Metaphorical Structuring View, Spatial Cuing Paradigm, Attentional Orienting

\section{Introduction}

Due to the non-substantiality and abstractness of temporal concepts, people generally think about these abstract concepts by associating with more concrete concept, such as spatial orientation (Haspelmath, 1997; Radden, 2011). In many languages, the spatial-temporal conceptual metaphor is widely used, e.g., "back 
in the 60s", "in the weeks ahead of us" (Clark, 1973; Lakoff \& Johnson, 1980b). The expression of temporal concepts differs across languages. Previous studies have shown that English speakers use the horizontal spatial-temporal metaphor (e.g., "to look back 20 years" to indicate the past). In contrast with English, Chinese native speakers express temporal concept by using vertical metaphor (Boroditsky, 2011; Boroditsky, Fuhrman, \& McCormick, 2011; Fuhrman \& Boroditsky, 2010; Fuhrman et al., 2011). For example, “shang4 xing1 qi1” (上星期) indicates “last week" and “xia4 xing1 qi1” (下星期) means “next week", in which the Chinese character "shang4" (上) stands for "top or upper", the character “xia4" (下) for "bottom or lower". If linguistic differences affect conceptualization of temporal concepts, it is likely that speakers across different languages will have different mechanisms behind the spatial-temporal metaphor. Considering the significant divergence in temporal expressions between English and Chinese, exploring spatial-temporal metaphors in Chinese is necessary. In the present study, we examined the mechanism of multi-dimensional spatial-temporal metaphors in Chinese speakers.

A number of studies have reported horizontal spatial-temporal metaphor in English (Boroditsky, 2011; Boroditsky et al., 2011; Fuhrman et al., 2011; Ouellet, Santiago, Jesus Funes, \& Lupianez, 2010; Torralbo, Santiago, \& Lupiáñez, 2006). For example, Torralbo et al. (2006) using space-time congruency effect to address the existence of mental timeline that runs from left to right. However representations of linguistic notations differ across languages (Hung, Hung, Tzeng, \& Wu, 2008). Hung et al. (2008) found that different notations of the same concept have flexible mappings within space, influenced by the dominant contexts. They examined the orientation of the mental number line for different numerical notations (e.g., “1”, “一”, “壹” all mean one) in Chinese speakers. Results showed that Arabic numerals are mentally aligned horizontally, while Chinese number words are aligned vertically. If abstract notations, such as numerical representation, may have different representations in different cultures, we assume that the concept of time can also be influenced by culture. How these processes interact in non-alphabetic language, like Chinese, remains unclear.

Boroditsky (2001) found that Chinese speakers tended to use vertical spatial-temporal metaphor even when they processed temporal concept in English. Participants need to answer some questions about time (e.g. March comes earlier than April.) after processing a horizontal or vertical array of objects. The results showed that Chinese speakers response faster after the priming of vertical arrays. In an additional experiment, native English speakers were trained to talk about time using vertical spatial expressions, then their responses to the temporal became closer to those of Chinese speakers. Therefore, the results from Boroditsky's studies supported that Chinese speakers tend to think about time vertically, and different languages shape abstract concept differently.

However, Chen (2007) failed to replicate Boroditsky (2001)'s results, and it suggested that Chinese speakers actually use horizontal spatial metaphors more 
often than vertical metaphors. Chen utilized the Yahoo search engine and Google News Taiwan to estimate the frequencies of using horizontal and vertical spatial metaphors when Chinese people expressed time. Results clearly showed that horizontal spatial metaphors were used more frequently than vertical spatial metaphors in Chinese speakers. This finding was inconsistent with the results of Boroditsky (2001). Therefore, Chen concluded that, compared to the English speakers, Chinese speakers do not think about time in a different way, although Chinese speakers use vertical spatial metaphor to express time.

Different tasks and stimuli might cause these inconsistent results. For example, Boroditsky (2001) adopted spatial priming paradigm. In this paradigm, the participants were asked to observe the objects in a horizontal or vertical array, and their reaction times to the temporal relationship judgments were recorded. While this paradigm made participants represent spatial concepts before they process the concept of time. Therefore, this paradigm leads participants to use different spatial frame during the temporal relationship processing. It can not clearly prove the relationships between temporal and spatial representations. Furthermore, although Chen (2007) made some changes in the experimental materials, their participants were Chinese-English bilinguals. English experiences influence their conceptualization even when they are manipulating Chinese. In the present study, though spatial cuing paradigm (Posner, 1980; Posner, Rafal, Choate, \& Vaughan, 1985; Ouellet, Santiago, Jesus Funes, \& Lupianez, 2010), we were interesting in examining the psychological impact of the Chinese vertical and horizontal spatial-temporal metaphor, and exploring the dominant dimensions between the two metaphors processing under the Chinese context.

In spatial cuing paradigms, attention is cued to one area of the computer screen, and the responses are influenced by the cue stimulus. Centrally and peripherally presented cues can orient attention (Posner \& Cohen, 1984). Ouellet et al. (2010) used cuing paradigm to demonstrate that temporal words can orient spatial attention. In the present study, participants needed to finish a localization task. Chinese temporal words were utilized in this study to determine whether these words can orient spatial attention or not.

In order to fill these important gaps in the research of temporal-spatial metaphor, current study was conducted to solve three questions as following. 1) in the processing of temporal concepts, do Chinese speakers use the horizontal spatial-temporal metaphor? In experiment 1 , we adopted a new paradigm to replicate the findings that horizontal spatial-temporal metaphor exists in Chinese speakers (Boroditsky, 2000, 2001). 2) In the processing of temporal concepts, do Chinese speakers also use vertical spatial-temporal metaphor? Based on many previous studies (Boroditsky, 2000, 2001; Boroditsky, 2011; Boroditsky et al., 2011; Bottini \& Casasanto, 2010; Casasanto \& Boroditsky, 2008; Casasanto \& Bottini, 2010; Fuhrman \& Boroditsky, 2010; Gentner et al., 2002; Lakoff \& Johnson, 1980a, 1980b; Merritt et al., 2010; Scott, 1989), we assumed that Chinese speakers use the vertical spatial-temporal metaphor. 3) If Chinese speakers both have vertical and horizontal metaphors, which one will be the dominant dimension? 
We also explored the relationship between these two metaphors.

\section{Experiment 1}

The goal of Experiment 1 was to test whether Chinese temporal reference modulate processing in a concurrent horizontal localization task for speakers of Chinese.

\subsection{Method}

\subsubsection{Participants}

Twenty-seven young adults (mean age $=21$ years; 12 females and 15 males) participated in Experiment 1. All the participants were right-handed native speakers of Chinese with normal (or corrected) vision and no reports of neurological disorders. All the participants had passed the national mandarin proficiency test and attained the certificate of level 2 or above, which means they have a relatively high level of Chinese proficiency. As well, all participants gave written informed consent in accordance with the guidelines of the Human Subjects Committee of South China University.

\subsubsection{Materials and Apparatus}

We selected 80 Chinese temporal words from Contemporary Chinese Dictionary (2005). All of the words were dissyllables and none of them contained spatial characters. For example, some temporal words such as "qian2 tian1" (“前天” means the day before yesterday) was not used because the character "qian2" (“前” means in front of something in Chinese) had the meaning of space. Forty-two college students rated each word from 1 to 5 (" 1 " indicated it was highly consistent with its meaning, and " 5 " indicated it was highly inconsistent with its meaning) to evaluate its temporal reference. After the evaluation, 48 Chinese temporal words were selected (see Appendix Table A1), Half of the words were past-related (the mean score of the past-related words is 4.60), such as "xil ri4" (“昔日” means in past days) and the other half referring to future time (the mean score of the future-related words is 4.24), such as “ming2 tian1” (“明天” means tomorrow or in the following days). All the words are located in the Appendix.

All words were presented in $24 \mathrm{pt}$. Songti font. The task was programmed in E-prime (Schneider, Eschman, \& Zuccolotto, 2002) and conducted on a computer using an Intel Pentium IV PC $1.70 \mathrm{GHz}$. Stimuli were presented on a 15 inch $(38.1 \mathrm{~cm})$ color monitor. The target was a dot of $5 \mathrm{~mm}$ diameter, which appeared in one of two $1.3 \times 1.3 \mathrm{~cm}^{2}$ boxes. The boxes were on both side of the screen $\left(7.39^{\circ}\right.$ of visual angle; $7.75 \mathrm{~cm}$ from the center). All stimuli were presented in white on a black background. The experiment was run in a sound-attenuated, dimly illuminated room.

\subsubsection{Procedure and Design}

Participants sat in a quiet room at approximately $60 \mathrm{~cm}$ from the screen. Figure 1 illustrated the procedure for Experiment 1. First, a fixation point was presented 


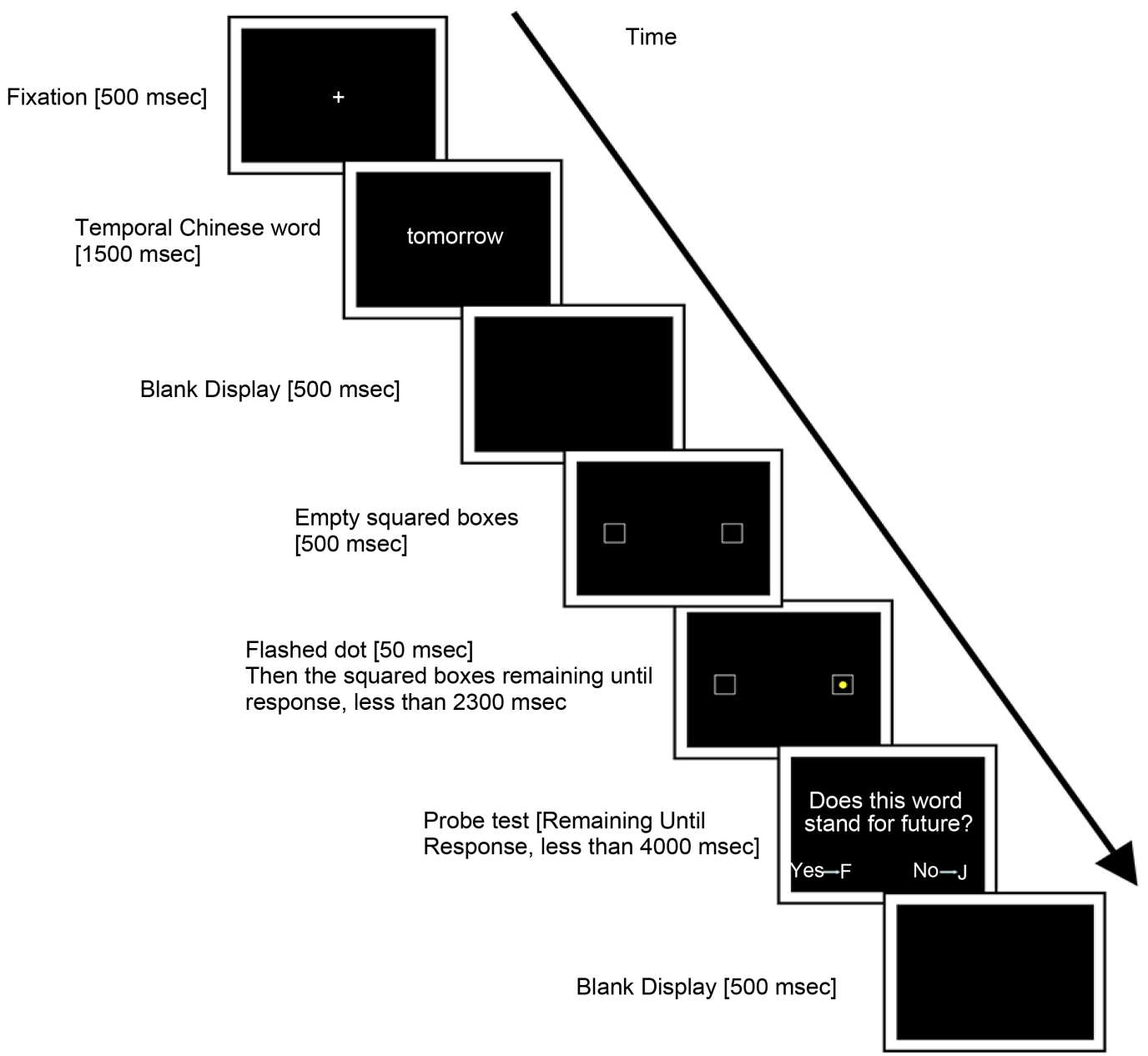

Figure 1. Event sequence of one trial in Experiment 1.

for $500 \mathrm{~ms}$, followed by a centrally presented past-related or future-related Chinese word for $1500 \mathrm{~ms}$. Participants were instructed to memorize the temporal reference of the presented words. A blank screen was presented for $500 \mathrm{~ms}$, and two empty square boxes were presented at the left and right positions of the monitor. After $250 \mathrm{~ms}$, a yellow dot flashed for $50 \mathrm{~ms}$ in one of the two boxes. The two boxes remained on the screen until the participant responded and timed out at $2300 \mathrm{~ms}$. At this point, participants performed the localization tasks to locate where the dot appeared. The participant should press the "F" key if the dot appeared to the left and the " $J$ " key if it appeared to the right.

In order to make sure that participants kept that temporal representation during the whole trial, after the localization task, a question was set for asking participants whether the word that just appeared refers to the past time or future. Participants were asked to press the "F" or "J" key to indicate a yes or no response. Participants were allowed $4000 \mathrm{~ms}$ to give their response, with longer latencies being excluded. Between trials a lank screen was presented for $1000 \mathrm{~ms}$. They were not told about any possible relationship between the cue word and the target location or the target location and the final probe question. 
The experiment had two blocks, differing in the mapping of " $F$ " and "J" to yes or no responses. The order of blocks was counterbalanced over participants. Within each block, each experimental word was presented four times, paired with targets at either location, and were presented with both final probe questions. Items across different conditions were presented in a random order. Participants were allowed to take a break between blocks. Each block consisted of 16 practices and 192 experimental trials. The experiment lasted about 45 - $50 \mathrm{mi}$ nutes.

\subsection{Results and Discussion}

The results of reaction time in different conditions are summarized in Table 1. Localization errors were $0.43 \%$ of trials, and memory errors were $6.7 \%$ of trials.

The latency data were submitted to a 2 (Temporal Reference: past/future) $\times 2$ (Target Location: left/right) two-way repeated measures ANOVA. In the latency analysis, the main effect of temporal reference was not significant, $F(1,26)=$ $0.23, \mathrm{MSE}=126.49$. The main effect of target location was also not significant, $F(1,26)=0.02, \operatorname{MSE}=6.80$. However, we found a significant interaction between temporal reference and target location, $F(1,26)=6.50$, MSE $=268.23, p=0.017$.

The simple effect test revealed that when the targets were on the left side, there was a significant difference between different types of temporal reference, $F(1,26)=6.40, \mathrm{MSE}=173.23, p=0.018$, which indicates that the participants responded faster to the past-related words than the future-related words. When the targets were on the right side, there was no significant difference between different temporal references in the subject analysis, $F(1,26)=2.99$, MSE $=$ 221.49, $p=0.096$, but there was a trend that participants responded faster to the future-related words than the past-related words from descriptive results.

The results in Experiment 1 showed a left-past/right-future (horizontal) facilitating effect for the targets presented at the location cued by the centrally presented Chinese temporal words. Specifically, when the targets were on the left side, the responses to the past-related words were significantly faster than the future-related words, and vice versa. This suggested that temporal reference is able to orient attention along the horizontal axis in Chinese speakers. Further, consistent with Ouellet et al. (2010), this result supported that Chinese speakers use spatial-temporal metaphor representation in the horizontal axis (i.e., the "left-past/right-future" phenomenon).

Table 1. Means and standard deviations (in Parentheses) of the Latency (ms) per Condition in Experiment 1 for temporal reference and target location.

\begin{tabular}{ccc}
\hline Temporal reference & Past-related & Future-related \\
\hline Target location & & \\
Left & $274(57)$ & $283(67)$ \\
Right & $281(72)$ & $274(61)$ \\
\hline
\end{tabular}




\section{Experiment 2}

In Experiment 1, we found evidences that the horizontal spatial-temporal metaphor exists in Chinese speakers. However, it is still unclear whether this is the only dimension used or not. The purpose of Experiment 2 was to investigate whether there was a vertical dimension that Chinese speakers also use when they represent time.

\subsection{Method}

\subsubsection{Participants}

Twenty-eight native Chinese speaking adults (mean age $=23$ years; 13 females and 15 males) participated in this experiment.

\subsubsection{Materials and Apparatus}

The same words in Experiment 1 were used.

\subsubsection{Procedure and Design}

The procedure in Experiment 2 is similar to Experiment 1. The only difference is that the dimension was vertical rather than horizontal (i.e., the " 7 " and " $n$ " keys). In order to balance the facilitating effect that right-left hand response to up-down localization may cause, half of the participants used their left hand to press the top key and their right hand to press the bottom key, and vice versa for the other half. Both the order of the responding-key assignment and the hand position were counterbalanced across participants.

Participants were allowed to take a break between blocks. Each block consisted of 16 practice and 192 experimental trials. The experiment lasted about 45 to 50 minutes.

\subsection{Results and Discussion}

Out of the correct trials, $4.36 \%$ of trials were outliers (3 SD above or below each participant's mean) and were discarded from the reaction time analyses. The results are summarized in Table 2.

The latency data were conducted to a 2 (temporal reference: past-related/ future) $\times 2$ (target location: top/bottom) two-way repeated measure ANOVA. In the latency analysis, the main effect of temporal reference was not significant, $F(1,27)=0.019, \mathrm{MSE}=256.78$. The main effect of target location also was not significant, $F(1,27)=0.086, \mathrm{MSE}=967.36$. However, again we found a significant interaction between temporal reference and target location, $F(1,27)=$

Table 2. Means and standard deviations (in Parentheses) of the Latency (ms) per Condition in Experiment 2 for temporal reference and target location.

\begin{tabular}{ccc}
\hline Temporal reference & Past-related & Future-related \\
\hline Target location & & \\
Top & $304(36)$ & $312(42)$ \\
Bottom & $312(41)$ & $306(35)$ \\
\hline
\end{tabular}


14.715, MSE $=90.64, p=0.001$.

The simple effect test demonstrated that, when the targets were located on the top, a significant difference between different types of temporal reference was found, $F(1,27)=4.35$, MSE $=172.99, p=0.047$, suggesting that the participants responded faster to the past-related words than the future-related words; when the targets were placed at the bottom, there was also a marginally significant difference between different temporal references, $F(1,27)=3.39$, MSE $=174.48, p=$ 0.076 , suggesting that participants responded faster to the future words than the past words.

The results in Experiment 2 showed a top-past/bottom-future (vertical) facilitating effect for targets presented at the location cued by the centrally presented Chinese temporal words. Specifically, when the targets were at the top, the responses to the past-related words were significantly faster than that for the future-related words. When the targets were at the bottom, the responses to the past-related words were significantly slower than that for the future-related words. This indicated that temporal reference was able to orient attention along the vertical axis for Chinese speakers.

\section{Experiment 3}

The results from Experiment 1 and Experiment 2 demonstrated that Chinese speakers used both vertical and horizontal spatial-temporal metaphors when they represented Chinese temporal words. A further question arises that if Chinese speakers use both vertical and horizontal spatial-temporal metaphors, which one would dominate when they processed temporal information? Therefore, Experiment 3 was designed to explore which spatial metaphor was the dominant factor between the two dimensions.

\subsection{Method}

\subsubsection{Participants}

Twenty-eight native Chinese speaking adults (mean age $=22.7$ yrs., 10 females and 18 males) participated this experiment.

\subsubsection{Materials and Apparatus}

The same words as in Experiment 1 and 2 were used.

\subsubsection{Procedure and Design}

Except for one important difference, the procedure in Experiment 3 was similar to Experiments 1 and 2. In Experiment 3, the dimension was changed from a single dimension to dual dimensions (right-left and top-bottom). Using a numerical key pad, the participants pressed the number keys " 8 " (top), " 2 " (bottom), " 4 " (left), or " 6 " (right) to respond to the positions of flashed dots on the screen. To avoid the interference from the original button position in the final probe question, the participants orally responded "Yes" or "No" to the temporal question, for examples, "Does the word presented right now refer to the past 
time?" The experimenter recorded whether the answer was right or wrong. Then the participants pressed the key " 5 " to continue the task. Participants were allowed to take a break between blocks. Each block consisted of 16 practices and 192 experimental trials.

\subsection{Results and Discussion}

Table 3 showed the mean of the latency data in Experiment 3. The latency data were conducted to a 2 (temporal reference: past/future) $\times 2$ (target dimension: vertical axis/horizontal axis) two-way repeated measure ANOVA. In the latency analysis, the main effect of temporal reference was not significant, $F(1,27)=$ $0.15, \mathrm{MSE}=70.82$. The main effect of target dimension was significant, $F(1,27)=$ 12.47, MSE $=1746.57, p=0.002$. More importantly, a significant interaction between temporal reference and target dimension was observed, $F(1,27)=11.00$, MSE $=190.56, p=0.003$.

The simple effect test showed that when the temporal words indicate past time, there was a significant difference in the different dimensions, $F(1,27)=$ 20.71, MSE $=902.55, p<0.001$, that is, the participants responded faster to the horizontal dimension than to the vertical dimension. When the temporal words indicated future time, there was also a significant difference for the different dimensions, $F(1,27)=5.01, \mathrm{MSE}=1034.04, p=0.034$, showing that participants responded faster to the horizontal dimension than to the vertical dimension.

Furthermore, the latency data were also submitted to a 2 (temporal reference: past/future) $\times 4$ (target position: top/bottom/right/left) two-way repeated measure ANOVA. The results showed that the main effect of temporal reference was not significant, $F(1,27)=0.15, \mathrm{MSE}=141.65$. The main effect of target position was significant, $F(1,27)=12.04, \mathrm{MSE}=1939.52, p<0.001$. More importantly, we found a significant interaction between temporal reference and target position, $F(1,27)=6.05, \mathrm{MSE}=354.51, p=0.001$.

The simple effect test results showed when the temporal words described past time, there was a significant difference among target positions, $F(3,81)=18.23$, MSE $=1075.41, p<0.001$, furthermore, the participants responded faster to the past-related targets on the left than that on the top. When the temporal words described future time, there was also a significant difference among target positions, $F(3,81)=4.84, \mathrm{MSE}=1217.74, p=0.004$, and the participants responded

Table 3. Means and standard deviations (in Parentheses) of the Latency (ms) per Condition in Experiment 3 for temporal reference and target location.

\begin{tabular}{ccc}
\hline Temporal reference & Past-related & Future-related \\
\hline Target location & & \\
Left & $386(100)$ & $399(113)$ \\
Right & $396(112)$ & $399(111)$ \\
Up & $408(105)$ & $407(106)$ \\
Down & $446(122)$ & $429(106)$ \\
\hline
\end{tabular}


faster to the future-related target on the right than that under the bottom.

The results of Experiment 3 showed a facilitating effect for the targets presented at the location cued by past-related and future-related Chinese temporal words. Specifically, when the stimuli were past-related temporal words, the responses to the left were significantly faster than to those at the top. When the stimuli were future temporal words, the responses to the right were significantly faster than at the bottom. This suggested that temporal reference was able to orient attention in Chinese speakers, and that the horizontal dimension was more dominant than the vertical dimension.

\section{General Discussion}

The results of Experiments 1 and 2 demonstrated that Chinese temporal words induced visuo-spatial attentional orienting for Chinese speakers. Past-related temporal words were able to orient attention to the left or top, and the future temporal words were able to orient attention to the right or bottom. The results of Experiment 3 demonstrated that Chinese speakers tended to respond faster in the horizontal dimension than in the vertical dimension in the processing of Chinese temporal words. This signified that temporal reference is able to orient attention in Chinese speakers and the horizontal axis is the dominant dimension compared to the vertical axis. These findings also demonstrated that the representation of time depends on the representation of space, supporting the Metaphorical Structuring View (Lakoff \& Johnson, 1980a).

These results are partially inconsistent with one of the conclusions by Boroditsky (2001) which English speakers used horizontal spatial-temporal metaphor to represent time, while Chinese speakers used vertical spatial-temporal metaphor instead. In Boroditsky's study, the spatial priming paradigm was used, which prompted the spatial orientation to activate before the time concepts were processed. Therefore, the results could not strongly demonstrate that people represent the concept of time by using spatial concepts. Moreover, all the participants in that study were Chinese-English bilinguals, and all the materials were in English. The processing of temporal words in Chinese might be influenced by English language and their English thinking style (Luna, Ringberg, \& Peracchio, 2008). In the current study, the participants processed the time concepts and then made the judgment of spatial localizations.

The present study also indicated that the horizontal dimension is the dominant one in Chinese spatial-temporal metaphor, which is in accord with many previous studies (Boroditsky, 2011; Boroditsky et al., 2011; Chen, 2007; Scott, 1989). This could be due to writing direction, as Tversky, Kugelmass, and Winter (1991) asked Arabic and English speaking participants to arrange chronological order of events in horizontal direction (such as three meals a day in order). Arabic is written in the opposite direction to English, namely from right to left. In that study, the results showed that British English speaking participants tended to arrange the events in the direction from left to right, while the Arabic 
speakers arranged the events from right to left direction. It showed that the responses of English and Hebrew speakers were congruent with their writing directionality. These results suggest that, when people represent the temporal concepts, the participants automatically access their cultural specific spatial representations even when space is implicit to the task.

Based upon these findings, we assumed that the writing direction affects the way of representing time, and the dominant dimension is affected by the Chinese right-to-left writing habits. In China, from ancient times until around 1955, vertical direction was the standard orientation of written text, and people can get to these written works in daily life. Therefore, this historical orientation may be one of the factors that influence Chinese speakers to have the vertical spatial-temporal metaphor. For contemporary Chinese speakers, the frequency of writing and reading in horizontal direction is much higher than that in vertical direction, though a few reading materials are still written in vertical direction. Therefore, the dominant dimension is the horizontal dimension in most common reading tasks in Chinese, and may be part of the reasoning as to why the horizontal dimension is more pronounced.

The phenomenon that Chinese speakers use the vertical dimension of space concepts to characterize time also can due to linguistic factors, such as the influence from native language, culture, and Chinese history. The vertical temporal reference emerged in Chinese literature thousands of years ago. Lan (1999) pointed out that the vertical temporal reference in Chinese was a concept purely based on spatial reference and was found in the earliest known stage of the development of written Chinese. And this temporal-spatial metaphor could possibly be related to the movement of the sun. In addition, it might also have a relationship with the traditional Chinese culture. In ancient China, most people place the ancestors' name boards on the high desks, and they kneeled down to worship ancestors. Base on these customs, the ancestors in China also are called as /Shang4 Zu3/ (/Shang4/ means "up", /Zu3/ means "generation"), and the younger generations are called as /Xia4 Bei4/ (/Xia4/ means "down", /Bei4/ also means "generation"), which contains the spatial reference "up" and "down".

The psychological impact and processing mechanism of the spatial-temporal conceptual metaphor had been a controversial topic in recent years (Boroditsky, 2000, 2001; Boroditsky, 2011; Boroditsky et al., 2011; Bottini \& Casasanto, 2010; Casasanto \& Boroditsky, 2008; Casasanto \& Bottini, 2010; Chen, 2007; Fuhrman \& Boroditsky, 2010; Gentner et al., 2002; Lakoff \& Johnson, 1980b; Ouellet et al., 2010; Torralbo et al., 2006; Tversky \& Shafir, 2004; Tversky \& Franklin, 1994). The present study provided evidence supporting the Metaphorical Structuring View (Lakoff \& Johnson, 1980a) in many aspects. According to this view (McGlone \& Harding, 1998; Gentner, Imai, \& Boroditsky, 2002), people use spatial representations to describe temporal representations. It has been shown that the development of human spatial perception occurs much earlier than that of time perception (Zhou \& Huang, 2001). Therefore, it can be reasonable that 
people use spatial perception to represent time, which also can be found in early developmental stages of children. Furthermore, people have a stronger perception of movement and space, but relatively have a weaker perception of time. Due to the constraints of the physiological basis of this phenomenon, people use spatial perception to represent time.

Because of the spatial-temporal conceptual metaphor, humans can imagine an invisible time event into a cognitive system in reality. Therefore, the studies in this area explored the spatial representation of the metaphor of time to reveal the nature of time thinking and time perception. This exploration can help us to understand the information of the concept of time, and the representation of the time concept model, as well as the effects of language and culture on the representation of concepts.

\section{Acknowledgements}

This research was supported by National Natural Science Foundation of China (31671132) and the MOE Project of Key Research Institute of Humanities and Social Sciences in Universities (15JJD190005).

\section{Contribution}

We state that all the authors and co-authors have given substantial contribution for this manuscript.

\section{References}

Boroditsky, L. (2000). Metaphoric Structuring: Understanding Time through Spatial Metaphors. Cognition, 75, 1-28. https://doi.org/10.1016/S0010-0277(99)00073-6

Boroditsky, L. (2001). Does Language Shape Thought?: Chinese and English Speakers' Conceptions of Time. Cognitive Psychology, 43, 1-22. https://doi.org/10.1006/cogp.2001.0748

Boroditsky, L. (2011). How Language Shapes Thought. Scientific American, 304, 62-65. https://doi.org/10.1038/scientificamerican0211-62

Boroditsky, L., Fuhrman, O., \& McCormick, K. (2011). Do English and Chinese Speakers Think about Time Differently? Cognition, 118, 123-129. https://doi.org/10.1016/j.cognition.2010.09.010

Bottini, R., \& Casasanto, D. (2010). Implicit Spatial Length Modulates Time Estimates, But Not Vice Versa. In C. Hölscher, T. F. Shipley, M. Olivetti Belardinelli, J. A. Bateman, \& N. Newcombe (Eds.), Spatial Cognition VII. International Conference, Spatial Cognition 2010, Mt. Hood/Portland, OR, 15-19 August 2010, 152-162. Berlin Heidelberg: Springer. https://doi.org/10.1007/978-3-642-14749-4_15

Casasanto, D., \& Boroditsky, L. (2008). Time in the Mind: Using Space to Think about Time. Cognition, 106, 579-593. https://doi.org/10.1007/978-3-642-14749-4_15

Casasanto, D., \& Bottini, R. (2010). Can Mirror-Reading Reverse the Flow of Time? In C. Hölscher, T. F. Shipley, M. Olivetti Belardinelli, J. A. Bateman, \& N. S. Newcombe (Eds.), Spatial Cognition VII. International Conference, Spatial Cognition 2010, Mt. Hood/Portland, OR, 15-19 August 2010, 335-345. Berlin Heidelberg: Springer. https://doi.org/10.1007/978-3-642-14749-4_28 
Chen, J. (2007). Do Chinese and English Speakers Think about Time Differently? Failure of Replicating Boroditsky (2001). Cognition, 104, 427-436. https://doi.org/10.1016/j.cognition.2006.09.012

Clark, H. H. (1973). Space, Time, Semantics, and the Child. Cognitive Development and the Acquisition of Language, 27, 63.

Fuhrman, O., \& Boroditsky, L. (2010). Cross-Cultural Differences in Mental Representations of Time: Evidence from an Implicit Nonlinguistic Task. Cognitive Science, 34, 1430-1451. https://doi.org/10.1111/j.1551-6709.2010.01105.x

Fuhrman, O., McCormick, K., Chen, E., Jiang, H., Shu, D., Mao, S., \& Boroditsky, L. (2011). How Linguistic and Cultural Forces Shape Conceptions of Time: English and Chinese Time in 3D. Cognitive Science, 35, 1305-1328. https://doi.org/10.1111/j.1551-6709.2011.01193.x

Gentner, D., Imai, M., \& Boroditsky, L. (2002). As Time Goes by: Evidence for Two Systems in Processing Space-Time Metaphors. Language and Cognitive Processes, 17, 537-565. https://doi.org/10.1080/01690960143000317

Haspelmath, M. (1997). From Space to Time: Temporal Adverbials in the World's Languages. Lincom Europa.

Hung, Y, Hung, D. L., Tzeng, O. J. L., \& Wu, D. H. (2008). Flexible Spatial Mapping of Different Notations of Numbers in Chinese Readers. Cognition, 106, 1441-1450.

Lakoff, G., \& Johnson, M. (1980a). Conceptual Metaphor in Everyday Language. The Journal of Philosophy, 77, 453-486. https://doi.org/10.2307/2025464

Lakoff, G., \& Johnson, M. (1980b). Metaphors We Live by. Chicago: University of Chicago Press.

Lan, C. (1999). Chinese Spatial Metaphor in Cognitive Aspect. Foreign Language Teaching and Research, 4, 7-15. (In Chinese)

Luna, D., Ringberg, T., \& Peracchio, L. (2008). One Individual, Two Identities: Frame Switching among Biculturals. Journal of Consumer Research, 35, 279-293.

https://doi.org/10.1086/586914

McGlone, M. S., \& Harding, J. L. (1998). Back (or Forward?) to the Future: The Role of Perspective in Temporal Language Comprehension. Journal of Experimental Psychology-Learning Memory and Cognition, 24, 1211-1223.

https://doi.org/10.1037/0278-7393.24.5.1211

Merritt, D. J., Casasanto, D., \& Brannon, E. M. (2010). Do Monkeys Think in Metaphors? Representations of Space and Time in Monkeys and Humans. Cognition, 117, 191-202.

Ouellet, M., Santiago, J., Jesus Funes, M., \& Lupianez, J. (2010). Thinking about the Future Moves Attention to the Right. Journal of Experimental Psychology-Human Perception and Performance, 36, 17-24. https://doi.org/10.1037/a0017176

Posner, M. I. (1980). Orienting of Attention. Quarterly Journal of Experimental Psychology, 32, 3-25. https://doi.org/10.1080/00335558008248231

Posner, M. I., \& Cohen, Y. (1984). Components of Visual Orienting. In H. Bouma, \& D. Bowhuis (Eds.), Attention and Performance $X$ (pp. 531-556). Hillsdale, NJ: Erlbaum.

Posner, M. I., Rafal, R. D., Choate, L. S., \& Vaughan, J. (1985). Inhibition of Return: Neural Basis and Function. Cognitive Neuropsychology, 2, 211-228. https://doi.org/10.1080/02643298508252866

Radden, G. (2011). Spatial time in the West and the East. Space and Time in Language, $1-40$.

Schneider, W., Eschman, A., \& Zuccolotto, A. (2002). E-Prime: User's Guide. Psychology Software Incorporated. 
Scott, A. (1989). The Vertical Dimension and Time in Chinese. Australian Journal of Linguistics, 9, 295-314. https://doi.org/10.1080/07268608908599424

Torralbo, A., Santiago, J., \& Lupiáñez, J. (2006). Flexible Conceptual Projection of Time onto Spatial Frames of Reference. Cognitive Science, 30, 745-757. https://doi.org/10.1207/s15516709cog0000_67

Tversky, A., \& Shafir, E. (2004). Preference, Belief, and Similarity: Selected Writings. Cambridge, MA: MIT Press.

Tversky, B., \& Franklin, N. (1994). Spatial Mental Models from Descriptions. Journal of the American Society for Information Science, 45, 656-668. https://doi.org/10.1002/(SICI)1097-4571(199410)45:9<656::AID-ASI3>3.0.CO;2-1

Tversky, B., Kugelmass, S., \& Winter, A. (1991). Cross-Cultural and Developmental Trends in Graphic Productions. Cognitive Psychology, 23, 515-557.

Zhou, R., \& Huang, X. (2001). A Study on Children's Development of the Capacity of Gaining Metaphorical Representation of Time. Psychological Science-SHANGHAI, 24, 606-609. (In Chinese)

\section{Appendix}

Table A1. Stimulus words with approximate literal translations.

\begin{tabular}{cccc}
\hline Past & Translation & Future & Translation \\
\hline 昔日 & in former days & 未来 & future \\
远古 & ancient times & 将来 & future \\
曾经 & once upon a time & 明年 & next year \\
往昔 & in the past & 来年 & next year \\
过去 & in the past & 来春 & next spring \\
昨天 & yesterday & 明天 & tomorrow \\
以往 & before & 明朝 & tomorrow \\
古代 & ancient times & 将要 & be going to \\
去年 & last year & 明晚 & next night \\
旧日 & former days & 明早 & next morning \\
旧时 & old times & 来日 & days to come \\
往日 & bygone days & 来世 & eternity \\
\hline
\end{tabular}


Submit or recommend next manuscript to SCIRP and we will provide best service for you:

Accepting pre-submission inquiries through Email, Facebook, LinkedIn, Twitter, etc. A wide selection of journals (inclusive of 9 subjects, more than 200 journals)

Providing 24-hour high-quality service

User-friendly online submission system

Fair and swift peer-review system

Efficient typesetting and proofreading procedure

Display of the result of downloads and visits, as well as the number of cited articles Maximum dissemination of your research work

Submit your manuscript at: http://papersubmission.scirp.org/

Or contact psych@scirp.org 